\title{
Effect of Direct Vertebral Rotation on the Spine Parameters (Coronal and Sagittal) in Adolescent Idiopathic Scoliosis
}

\section{Carlos Eduardo Gonçales Barsotti}

Hospital do Servidor Publico Estadual de Sao Paulo

Carlos Augusto Belchior B Junior

Hospital do Servidor Publico Estadual de Sao Paulo

\section{Rodrigo Mantelatto Andrade}

Universidade de Sao Paulo

Alexandre Penna Torini

Universidade de Santo Amaro

Ana Paula Ribeiro ( $\nabla$ apribeiro@usp.br)

Universidade de Sao Paulo Faculdade de Medicina https://orcid.org/0000-0002-1061-3789

Research article

Keywords: idiopathic scoliosis, adolescents, surgery, spine.

Posted Date: October 9th, 2020

DOl: https://doi.org/10.21203/rs.3.rs-88636/v1

License: (c) (i) This work is licensed under a Creative Commons Attribution 4.0 International License. Read Full License

Version of Record: A version of this preprint was published at Journal of Back and Musculoskeletal Rehabilitation on June 11th, 2021. See the published version at https://doi.org/10.3233/BMR-200320. 


\section{Abstract}

Background: Idiopathic scoliosis is accompanied by postural alterations, instability of gait, and functional disabilities. The objective was to verify radiographic parameters (coronal and sagittal) of adolescents with idiopathic scoliosis (AIS) pre- and post-surgery with direct vertebral rotation (DVR), associated with type 1 osteotomies in all segments (except the most proximal) and type 2 in the periapical vertebrae of the curves.

Methods: A prospective study design was employed in which 41 AIS were evaluated and compared preand post-surgery. Scoliosis was confirmed by a spine X-ray exam (Cobb angle). Eight radiographic parameters were measured: Cobb angles (thoracic proximal and distal), segmental kyphosis, total kyphosis, lumbar lordosis, pelvic incidence, sacral slope, and pelvic tilt.

Results: The Cobb angle averaged $51.3^{\circ} \pm 14.9^{\circ}$. Post-surgery, there were significant reductions for the following spine measurement parameters: Cobb angle thoracic proximal $(p=0.003)$; Cobb angle thoracic distal $(p=0.001)$; Cobb angle lumbar $(p=0.001)$; kyphosis (T5-T12, $p=0.012)$; and kyphosis (T1-T12, $p=0.002)$. These reductions showed the effectiveness of surgical correction to reduce Cobb angles and improve thoracic kyphosis. The values obtained for lumbar lordosis, pelvic incidence, sacral slope, and pelvic tilt were not significantly different pre- and post-surgery.

Conclusion: The surgical technique of DVR in AIS proved to be effective in the coronal and sagittal parameters directed at Cobb angles and thoracic kyphosis.

\section{Introduction}

Adolescent idiopathic scoliosis (AIS) is considered a three-dimensional spinal deformity most prevalent in children between the ages of 10 and 16 years in $80-90 \%$ of the cases [1-2]. The spine shows lateral curvature in the coronal plane, thoracic hypokyphosis in the sagittal plane, and intravertebral and intervertebral rotation in the transverse plane [3-4]. The ideal surgical procedure provides maximal correction and spinal balance with minimal fusion levels [4-6]. Three-dimensional deformities of both curves should be corrected [5].

We have used segmental pedicle screws with a spinal defeat maneuver in most scoliosis surgeries for the past two decades [4-7]. During the surgical correction of adolescent scoliosis, direct vertebral rotation (DVR) in combination with rod derotation after pedicle screw instrumentation enables correction of rotational vertebral body deformity, achieving a sufficient correction angle with a reduced fusion level while minimizing aggravated deformity and complications due to patient growth $[7,8]$. In the late 1990s, the literature showed use of the new method: DVR designed to foster rotational correction $[3,4,7-9]$. The choice of an inappropriate fusion level may result in under- or overcorrection of major and compensatory curves, in turn potentially causing serious problems such as trunk imbalance and decompensation [10]. 
A deformity of the right thoracic curve results in the apical and periapical vertebrae being rotated clockwise in the transverse plane $[10,11]$. To correct the intervertebral rotation, the direction of DVR should be opposite that of the rotational deformity, i.e., counter-clockwise in the transverse plane [11]. The direction of rod derotation (clockwise rotation) should be opposite that of DVR (counter-clockwise rotation) in the apical and periapical vertebrae of the right thoracic curve [8-10]. The direction of DVR in the lowest instrumented vertebra (LIV) and its effect on the uninstrumented curve are still undetermined $[4,10,11]$. It has been thought that the direction of DVR in the LIV might differ depending on the lumbar modifier, as described by Lenke et al. [12-14].

The authors of recent studies have focused on the radiologic outcomes of DVR in scoliosis surgery [914]. However, its efficacy and safety remain to be determined. Some suggest that DVR creates hypokyphosis on thoracic kyphosis, presents an increased risk of screw pullout, and prolongs operative time without benefits [15-17]. Surgical treatment can lead to improvements in self-confidence, selfimage, cosmetic and life satisfaction, and back pain [10-14]. Thoracic curves of $>50^{\circ}$ and the lumbar component of a double major curve will progress into adult life, especially in those with more apical rotation. Thoracolumbar curves do not affect pulmonary function but they do produce marked cosmetic deformity and increasing, although not disabling, back pain, often associated with a transitional shift of the vertebrae and a tendency to progress over time, often continuing after the end of spinal growth. Surgical treatment of these curves when they reach $50^{\circ}$ is therefore justified $[4,14]$.

The objective of the surgery in the treatment of AIS is to improve spine parameters and function with low complication rates and few long-term complications, according to the literature, especially in the DVR with the three-dimensional, corrective surgical technique $[15,16,17]$. However, due to the few studies on the specificity of this technique, little is understood about these parameters after surgical correction in patients with idiopathic scoliosis. Thus, the objective of this study was to verify radiographic parameters (coronal and sagittal) of AIS pre- and post-surgery with DVR associated with type 1 osteotomies in all segments (except the most proximal) and type 2 in the periapical vertebrae of the curves, according to the Schwab classification [18].

\section{Methods}

\section{Study Design and Participants}

This study was prospective and observational, and it included 41 AIS volunteer participants who were evaluated pre- and post-surgery. Recruitment was conducted through the Public Hospital in the State of Sao Paulo/SP, Brazil, between January 2018 and December 2019. The study procedure was reviewed and approved by the Departmental Research Committee of the Institute of Medical Assistance to the State Public Hospital Servant (registration number: 533.756), in accordance with the Helsinki Declaration and relevant guidelines and regulations. All participants our responsible parents provided their informed consent and then underwent radiographic assessment. 
All the patients with AIS were determined, radiographically, to have a single thoracic curve (Lenke 1-6) with Cobb angles of $51.3^{\circ} \pm 14.9^{\circ}$. The eligibility criteria were as follows: each participant must have had no history of deformity or pathology of the spine, hip, pelvis, or lower limbs; and each participant must have had no other musculoskeletal disorders, such as neuropathies, obesity, and/or rheumatoid arthritis, such as back pain for more than three consecutive months. In addition, they could not have prostheses and/or orthoses in the lower limbs (i.e., they had to have good general health), so as not to generate bias in the interpretation of pace evaluations [14-16].

\section{Radiographic Evaluations: Panoramic X-rays}

Full-length, free-standing spine radiographs with fists on clavicles were obtained in all subjects and measured by experienced radiation technologists. The radiographs were centered on T12 during inspiration, with a 2-meter distance between the film and the focus. All images were transferred to a computer as digital images and evaluated using the image software Surgimap Spine (Nemaris Inc., New York, USA) $[4,5,7,9]$.

Eight sagittal alignment and spinopelvic alignment parameters were analyzed on the radiographs of the 41 participants: Cobb angle thoracic proximal, Cobb angle thoracic distal, segmental kyphosis (T5-T12), total kyphosis (T1-T12), lumbar lordosis, pelvic incidence, sacral slope, and pelvic tilt. Segmental kyphosis was measured as the angle between the upper endplate of T5 and the lower endplate of T12; total thoracic kyphosis was measured using the T1 and T12 plateaus. Lumbar lordosis was measured using the angle formed between the upper endplate of L1 and S1. The pelvic incidence corresponded to the angle between the perpendicular to the upper S1 level passing through its center and the line connecting this point to the axis of the femoral head [19]. Sacral slope was defined as the angle formed by the upper endplate of $\mathrm{S} 1$ and the horizontal plane. Pelvic tilt was defined as the angle between the vertical plane and the straight line of the union between the femoral heads and the midpoint of the upper endplate of S1 [20].

\section{Surgical Procedure and Technique}

The posterior route with classic access in the midline was used. After subperiosteal dissection of the musculature, a Schwab type 1 osteotomy was performed at all levels of arthrodesis, except at the most proximal level and Schwab type 2 osteotomies in periapical vertebrae, according to the subjective assessment of the curve reducibility during the procedure [18]. All osteotomies were performed with a drill and Kerrison forceps (we did not use an osteotomy and hammer). Pedicle screws were used exclusively. The entry point and the insertion of uniplanar screws in the periapical and polyaxial vertebrae in the others was performed by anatomical parameters ("free-hand") [4]. All screws were checked by fluoroscopy; cobalt chrome rods were used and we did not use "cross-linking."

After placing the first hypermolded nail according to the patient's pelvic incidence, the block was defeated by the concave side of the deformity, followed by the placement of the second molded bar, according to the desired kyphosis, planned in the preoperative period. Then, direct vertebral defeat was performed at all levels (except for the neutral vertebrae) in the opposite direction of the rotation of the vertebra. In all 
cases, a suction drain was used, which was removed only at hospital discharge. Patients were encouraged to move early and orthotics were not prescribed in the postoperative period.

\section{Statistical Analysis}

The data were analyzed using SPSS version 20.0 (SPSS Inc., Chicago, IL). Calculation of the sample size was conducted based on the Cobb angle variable. A moderate effect size $(f=0.25)$, an $80 \%$ power, and a $5 \%$ significance level were used in the calculation. The baseline characteristics of participants were described as means and standard deviations. The means of the radiographic measurements were compared pre- and post-surgery using a Student's t-test. A significance level of $5 \%$ for two-tailed tests was considered as significant.

\section{Results}

Of the 41 volunteers, 13 were male and 28 were female. Their ages were $17.6 \pm 7.1$ pre-surgery and $18.8 \pm$ 7.2 post-surgery; Risser signs were $3.9 \pm 1.2$ pre-surgery and $4.3 \pm 0.8$ post-surgery.

Inter-observer reliability was high for eight spine parameters: 0.90 (Cobb angles); 0.89 (kyphosis angles); lumbar lordosis (0.93); pelvic incidence (0.91); sacral slope (0.92); and pelvic tilt (0.92). The measurements were considered as acceptable.

Table 1 shows the means and standard deviations found for the eight radiographic measurements preand post-surgery for all participants. The results show that post-surgery, there were significant reductions for the following spine measurement parameters: Cobb angle thoracic proximal ( $p=0.003)$; Cobb angle thoracic distal $(p=0.001)$; Cobb angle lumbar $(p=0.001)$; kyphosis (T5-T12, $p=0.012)$; and kyphosis (T1$T 12, p=0.002$ ). These reductions showed the effectiveness of surgical correction in reducing Cobb angles and thoracic kyphosis. The values obtained for lumbar lordosis, pelvic incidence, sacral slope, and pelvic tilt were not significantly different pre- and post-surgery. 
Table 1

Static posture plantar pressure distribution: means, standard deviations, and comparisons between groups (patients with AIS and the control).

\begin{tabular}{|llll|}
\hline Spine parameters (degree) & Pre-surgery & Post-surgery & $\mathbf{p}$ \\
\hline Cobb angle thoracic proximal (degree) & $20.7 \pm 9.1$ & $11.9 \pm 6.6$ & 0.003 \\
\hline Cobb angle thoracic distal (degree) & $51.3 \pm 14.9$ & $16.5 \pm 7.5$ & 0.001 \\
\hline Cobb angle lumbar (degree) & $44.0 \pm 16.0$ & $12.2 \pm 7.3$ & 0.001 \\
\hline Kyphosis (T5-T12, degree) & $23.4 \pm 12.5$ & $28.7 \pm 8.7$ & 0.012 \\
\hline Kyphosis (T1-T12, degree) & $35.9 \pm 11.8$ & $42.8 \pm 9.1$ & 0.002 \\
\hline Lumbar lordosis (degree) & $57.8 \pm 10.5$ & $57.0 \pm 11.0$ & 0.375 \\
\hline $\begin{array}{l}\text { Pelvic incidence (degree) } \\
\text { Sacral slope (degree) }\end{array}$ & $49.5 \pm 9.4$ & $49.1 \pm 9.2$ & 0.550 \\
\hline $\begin{array}{l}\text { Pelvic tilt (mm) } \\
\text { * Based on Student's t-test-dependent measures (pre- and post-surgery) } \text { considering differences of } p \\
<0.05 \text { as significant. }\end{array}$ & $4.1 \pm 9.0$ & $41.7 \pm 9.0$ & 0.419 \\
\hline
\end{tabular}

The Fig. 1 showed the improvement obtained by the surgical procedure with the DVR technique for the radiographic parameters of Cobb angle thoracic proximal (degree), Cobb angle thoracic distal (degree) and Cobb angle lumbar (degree). The Fig. 2 shows the improvement in thoracic kyphosis (T5-T12, degree) and no change in the parameters of lumbar lordosis in the patients evaluated.

\section{Discussion}

In this study we aimed to analyze the coronal and sagittal parameters after surgical treatment of AIS by the technique of DVR with type 1 and type 2 Schwab osteotomies. The main results showed that use of the technique yielded significant improvements in sagittal and coronal parameters, such as reductions in the proximal and distal Cobb angles as well as with the lumbar Cobb angle, in addition to an increase in segmental (T5-T12) and total thoracic kyphosis (T1-T12). The surgical technique for DVR is popular for correcting AIS, with efficacy on clinical and radiological parameters. However, the literature offers little with regard to its effectiveness in adolescents with AIS before and after the surgical procedure with DVR.

Using the DVR surgical technique associated with an osteotomy, a $68 \%$ correction of the main thoracic curve was observed in the coronal plane, with positive and significant improvement. This is similar to that observed in the study performed by Urbanski et al. [21], in which the authors evaluated 21 patients who underwent the DVR surgical procedure with researchers observing a $69 \%$ correction of the main thoracic curves. However, the divergence among related studies has been debated, especially with regard to sample standardization, surgical correction technique, and fixation materials used, as well as 
standardized surgery time for evaluations. These points lead to difficult comparisons. Thus, there is still a large divergence in post-surgical results using the DVR technique.

A recent meta-analysis carried out by Son et al. [22] has shown benefits with the DVR technique when compared to the simple spinal defeat technique. In this study, the association of the DVR technique with osteotomy was beneficial for increasing the correction of the thoracic curvature with an increase in kyphosis, but the risk-benefit of their choice must be weighed, given the possible post-surgical complications. Such care for surgical consideration is based on studies in which the authors did not find improvement in thoracic kyphosis using Schwab's type 2 osteotomy [23, 24, 25], but rather increased rates of bleeding during the procedure. Despite this, Seki et al. [11], using uniplanar screws, showed gains in correction of intervertebral rotation with the association of periapical Schwab type 2 osteotomies in relation to facetectomies, especially at lumbar levels. In this study, despite not having considered the parameters of rotational correction, we can observe benefits of performing osteotomy for the correction of thoracic kyphosis, in that it can benefit the patient's lung capacity.

Another important finding observed in this study was in the sagittal plane, with a considerable gain in segmental and total thoracic kyphosis, especially in patients classified by Lenke et al. [14] as hypokyphotic (eight patients with thoracic kyphosis T5-T12<10 $)$ with a preoperative average of $6.8^{\circ}$ of kyphosis to $20.1^{\circ}$ postoperatively. It is worth reiterating that all patients reached the normal range of kyphosis, according to the classification by Lenke et al. [14]. A study carried out in the past decade by Bernhart [26] revealed an important discussion about normality values for thoracic kyphosis (T3-T12) being between $9^{\circ}$ and $53^{\circ}$, while Stagnara [27] referred to the range of $7^{\circ}$ and $43^{\circ}$. The Spinal Deformity Study Group [28] offered a reference of $10^{\circ}$ to $40^{\circ}$ (T5-T12). Although the purpose of this study was not to verify normality parameters, the correction of the coronal and sagittal parameters among adolescents was positive, except for the parameters of lumbar lordosis, pelvic incidence, sacral slope, and pelvic tilt. These points can be explained by the DVR technique associated with osteotomy, since when correcting thoracic kyphosis, little change affects the region of the lumbar spine and pelvic segment. These findings are in agreement with the study by Urbanski et al. [21], in which the authors also did not observe significant changes in lumbar lordosis, but an increase in thoracic kyphosis with DVR in patients with AIS.

The use of DVR has been a source of disagreement regarding the maintenance or increase of thoracic kyphosis. Mladenov et al. [29] observed a decrease in thoracic kyphosis and lumbar lordosis in patients undergoing DVR compared to patients undergoing simple vertebral defeat. Urbanski et al. [21] showed improvement in the coronal plane with the DVR technique, but without differences in the sagittal plane compared to the group of patients submitted to simple defeat. Kim et al. [10], evaluating patients undergoing DVR, observed a lower number of arthrodesis and a lesser amount of intraoperative bleeding, despite not seeing significant differences in postoperative kyphosis in relation to the control group.

In a review study with meta-analysis, Son et al. [22] reported no significant differences in postoperative thoracic kyphosis between the groups undergoing DVR and those undergoing simple defeat. The differential of this study was to observe that perhaps the improvement of thoracic kyphosis was primarily 
due to type 2 osteotomy associating with the DVR technique, since none of the authors of the studies mentioned previously found significant increases in thoracic kyphosis using only the DVR technique.

One of the limitations of this study was that we did not consider postural parameters referring to the symmetry of the shoulders, nor did we consider the different types of AIS according to Lenke's classification. Consideration was not given to the rotation of the vertebrae in the pre- and postoperative periods or their implications for improving the quality of each patient's life and/or respiratory function.

The clinical relevance of this study points to the positive effect of the DVR technique associated with osteotomy in improving the parameters of thoracic kyphosis in patients with hypokyphotic predominance (T5-T12 < 10 ${ }^{\circ}$. According to Johnston et al. (2011) [30], hypokyphotic patients are associated with decreased lung function, especially in early onset curves. Still in this line of reasoning, Fuji et al. [31] observed an improvement in the pulmonary functioning of a patient with severe scoliosis (main thoracic curve of $96^{\circ}$ ) with hypokyphosis (T5-T12: $6^{\circ}$ ), with correction of the curve to $28^{\circ}$ in the coronal plane and to $14^{\circ}$ of kyphosis (T5-T12), while in this study we found $6.8^{\circ}$ of kyphosis which increased to $20.1^{\circ}$ in the postoperative period in hypokyphotic patients.

\section{Conclusion}

The surgical technique of direct vertebral defeat in adolescents with idiopathic scoliosis proved to be effective in reducing the coronal parameters and improving the sagittal parameters directed at Cobb angles and thoracic kyphosis, respectively. It is important to determine an appropriate treatment plan based on a more accurate assessment of clinical onset and prognosis.

\section{Abbreviations}

Adolescent idiopathic scoliosis (AIS); direct vertebral rotation (DVR); lowest instrumented vertebra (LIV).

\section{Declarations}

\section{Ethics approval and consent to participate}

This study was conducted in approved by the Departmental Research Committee of the Institute of Medical Assistance to the State Public Hospital Servant (number: 533.756), in accordance with the Helsinki Declaration and relevant guidelines and regulations and all patients had provided written informed consent prior to surgery.

\section{Consent for publication}

All patients had provided written informed consent for publication.

\section{Competing interests}


The authors declare that they have no competing interests.

\section{Funding}

Not applicable.

\section{Authors' contributions}

All authors were involved in drafting the article or revising it critically for important intellectual content, and all authors approved the final version to be published. Study conception, writing and design: CEGB, CABBJ and APR. Acquisition of data and Statistics: CEGB and APR. Analysis and interpretation of data: CABBJ, RMA, APT and APR.

\section{Acknowledgements}

The authors acknowledge for help and support of the all the participants and Spine Group of the Institute of Medical Assistance to the State Public Hospital Servant, in the state of Sao Paulo/SP, Brazil, during study.

\section{References}

1. Adobor RD, Rimeslatten S, Steen H, Brox JI. School screening and point prevalence of adolescent idiopathic scoliosis in 4000 Norwegian children aged 12 years. Scoliosis 2011;24(6):23. https://doi.org/10.1186/1748-7161-6-23.

2. Penha PJ, Ramos NLJP, de Carvalho BKG, Andrade RM, Schmitt ACB, João SMA. Prevalence of Adolescent Idiopathic Scoliosis in the State of São Paulo, Brazil. Spine, 2018;43(24):1710-1718. https://doi.org/10.1097/BRS.0000000000002725.

3. Lee SM, Suk SI, Chung ER. Direct vertebral rotation: a new technique of three-dimensional deformity correction with segmental pedicle screw fixation in adolescent idiopathic scoliosis. Spine 2004;29:343-349. https://doi.org/10.1097/01.brs.0000109991.88149.19.

4. Kim S, Kim J, Suk S. Effect of Direct Vertebral Rotation on the Uninstrumented Lumbar Curve in Thoracic Adolescent Idiopathic Scoliosis. Asian Spine J. 2017;11(1):127-137. https://doi.org/10.4184/asj.2017.11.1.127

5. Suk SI, Lee CK, Chung SS. Comparison of Zielke ventral derotation system and Cotrel-Dubousset instrumentation in the treatment of idiopathic lumbar and thoracolumbar scoliosis. Spine 1994;19:419-29. https://doi.org/ 1097/00007632-199402001-00007.

6. Suk SI, Lee CK, Kim WJ, Chung YJ, Park YB. Segmental pedicle screw fixation in the treatment of thoracic idiopathic scoliosis. Spine 1995,20:1399-405.

7. Huang Z, Wang Q, Yang J, et al. Vertebral derotation by vertebral column manipulator improves postoperative radiographs outcomes of Lenke $5 \mathrm{C}$ patients for follow-up of minimum 2 years. Clin Spine Surg 2016;29:E157-E161. https://doi.org/10.1097/BSD.0000000000000123. 
8. Okada E, Watanabe K, Pang L, et al. Posterior correction and fusion surgery using pedicle-screw constructs for Lenke type 5C adolescent idiopathic scoliosis: a preliminary report. Spine 2015;40:2530. https://doi.org/ 1097/BRS.0000000000000652.

9. Ries Z, Harpole B, Graves C, et al. Selective thoracic fusion of Lenke I and II curves affects sagittal profiles but not sagittal or spinopelvic alignment: a case-control study. Spine 2015;40:926-934. https://doi.org/ 1097/BRS.0000000000000861.

10. Kim GU, Yang JH, Chang DG, et al. Effect of Direct Vertebral Rotation in Single Thoracic Adolescent Idiopathic Scoliosis: Better 3-Dimensional Deformity Correction. World Neurosurg 2019;129:e401e408. https://doi.org/10.1016/j.wneu.2019.05.164.

11. Seki S, Kawaguchi Y, Nakano M, Makino H, Mine H, Kimura T. Rod rotation and differential rod contouring followed by direct vertebral rotation for treatment of adolescent idiopathic scoliosis: effect on thoracic and thoracolumbar or lumbar curves assessed with intraoperative computed tomography. Spine J. 2016;16(3):365-371. https://doi.org/10.1016/j.spinee.2015.11.032.

12. de Araujo FF, Marcon RM, Cristante AF, de Barros TEP, Letaif OB. Rotation assessment in adolescent idiopathic scoliosis with rod derotation. Acta Ortop Bras. 2019;27(1):42-45. https://doi.org/ 1590/1413-785220192701191874.

13. Sun L, Song Y, Liu L, An Y, Zhou C, Zhou Z. Bilateral apical vertebral derotation technique by vertebral column manipulation compared with vertebral coplanar alignment technique in the correction of Lenke type 1 idiopathic scoliosis. BMC Musculoskelet Disord 2013;14:175. https://doi.org/10.1186/1471-2474-14-175.

14. Lenke LG, Betz RR, Harms J, et al. Adolescent idiopathic scoliosis: a new classification to determine extent of spinal arthrodesis. J Bone Joint Surg Am. 2001;83:1169-81.

15. Bridwell KH. Surgical treatment of adolescent idiopathic scoliosis: the basics and the controversies. Spine 1994;19:1095-100. https://doi.org/ 1097/00007632-199405000-00020.

16. Krismer M, Bauer R, Sterzinger W. Scoliosis correction by Cotrel-Dubousset instrumentation: the effect of derotation and threedimensional correction. Spine1982;17:S263-9.

17. Di Silvestre M, Lolli F, Bakaloudis G, Maredi E, Vommaro F, Pastorelli F. Apical vertebral derotation in the posterior treatment of adolescent idiopathic scoliosis: myth or reality? Eur Spine J. 2013;22:31323. https://doi.org/ 1007/s00586-012-2372-2

18. Schwab F, Blondel B, Chay E, Demakakos J, Lenke L, Tropiano P, et al. The comprehensive anatomical spinal osteotomy classification. Neurosurgery 2014;74(1):112-20. https://doi.org/10.1227/NEU.0000000000000182o.

19. Legaye J, Duval-Beaupère G, Hecquet J, Marty C. Pelvic incidence: A fundamental pelvic parameter for three-dimensional regulation of spinal sagittal curves. Eur Spine J. 1998;7(2):99-103. https://doi.org/ 1007/s005860050038.

20. Le Huec JC, Hasegawa K. Normative values for the spine shape parameters using 3D standing analysis from a database of 268 asymptomatic Caucasian and Japanese subjects. Eur Spine $\mathrm{J}$ 2015;25(11):3630-7. https://doi.org/10.1007/s00586-016-4485-5

Page 10/13 
21. Urbanski W, Wolanczyk MJ, Jurasz W, Kulej M, Morasiewicz P, Dragan SL, et al. The impact of direct vertebral rotation (DVR) on radiographic outcome in surgical correction of idiopathic scoliosis. Arch Orthop Trauma Surg 2017;137(7):879-85. https://doi.org/ 1007/s00402-017-2700-4.

22. Son SM, Choi SH, Goh TS, Park W, Lee JS. Efficacy and Safety of Direct Vertebral Rotation in the Surgical Correction of Scoliosis: A Meta-Analysis. World Neurosurg 2019;124:e641-8. https://doi.org/ 1016/j.wneu.2018.12.170.

23. Halanski MA, Cassidy JA. Do multilevel ponte osteotomies in thoracic idiopathic scoliosis surgery improve curve correction and restore thoracic kyphosis? J Spinal Disord Tech 2013;26(5):252-5. https://doi.org/10.1097/BSD.0b013e318241e3cf.

24. Pizones J, Izquierdo E, Sánchez-Mariscal F, Álvarez P, Zúñiga L, Gómez A. Does wide posterior multiple level release improve the correction of adolescent idiopathic scoliosis curves? J Spinal Disord Tech 2010;23(7):24-30. https://doi.org/ 1097/BSD.0b013e3181c29d16.

25. Koerner JD, Patel A, Zhao C, Schoenberg C, Mishra A, Vives MJ, et al. Blood loss during posterior spinal fusion for adolescent idiopathic scoliosis. Spine 2014;39(18):1479-87. https://doi.org/ 1097/BRS.0000000000000439.

26. Bernhardt M, Bridwell KH. Segmental analysis of the sagittal plane alignment of the normal thoracic and lumbar spines and thoracolumbar junction. Spine 1989;14(7):717-721. https://doi.org/ 1097/00007632-198907000-00012.

27. Stagnara P, De Mauroy JC, Dran G, et al. Reciprocal angulation of vertebral bodies in a sagittal plane: approach to references for the evaluation of kyphosis and lordosis. Spine 1982;7(4):335-342. https://doi.org/ 1097/00007632-198207000-00003.

28. O'Brien M, Kulklo T, Blanke K, Lenke L. Radiographic Measurement Manual. Spinal Deform Study Gr Radiogr Meas Man 2008;120p.

29. Mladenov K V., Vaeterlein C, Stuecker R. Selective posterior thoracic fusion by means of direct vertebral derotation in adolescent idiopathic scoliosis: Effects on the sagittal alignment. Eur Spine $\mathrm{J}$ 2011;20(7):1114-7. https://doi.org/ 1007/s00586-011-1740-7.

30. Johnston CE, Stephens Richards B, Sucato DJ, Bridwell KH, Lenke LG, Erickson M. Correlation of preoperative deformity magnitude and pulmonary function tests in adolescent idiopathic scoliosis. Spine 2011;36(14):1096-102. https://doi.org/ 1097/BRS.0b013e3181f8c931.

31. Fujii T, Watanabe K, Toyama Y, Matsumoto M. Pulmonary function recovery demonstrated by ventilation-perfusion scan after posterior vertebral column resection for severe adolescent idiopathic scoliosis: A case report. Spine 2014;39(19):1190-4.

https://doi.org/10.1097/BRS.0000000000000458.

\section{Figures}



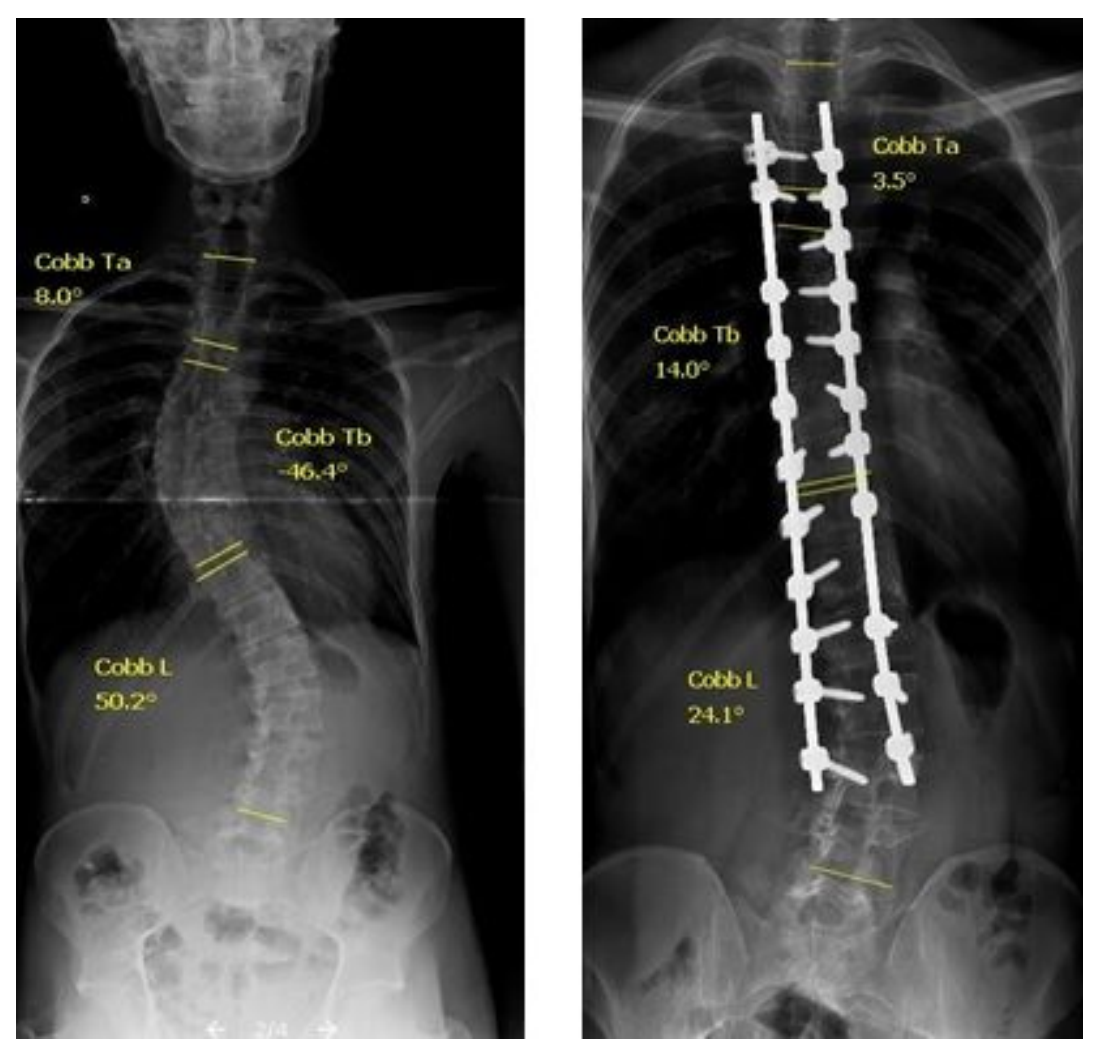

\section{Figure 1}

Representation of the improvement of radiographic parameters of the Cobb Angle proximal Thoracic (Cobb Ta), Cobb Angle distal Thoracic (Cobb Tb) and Cobb Angle Lumbar (Cobb L).
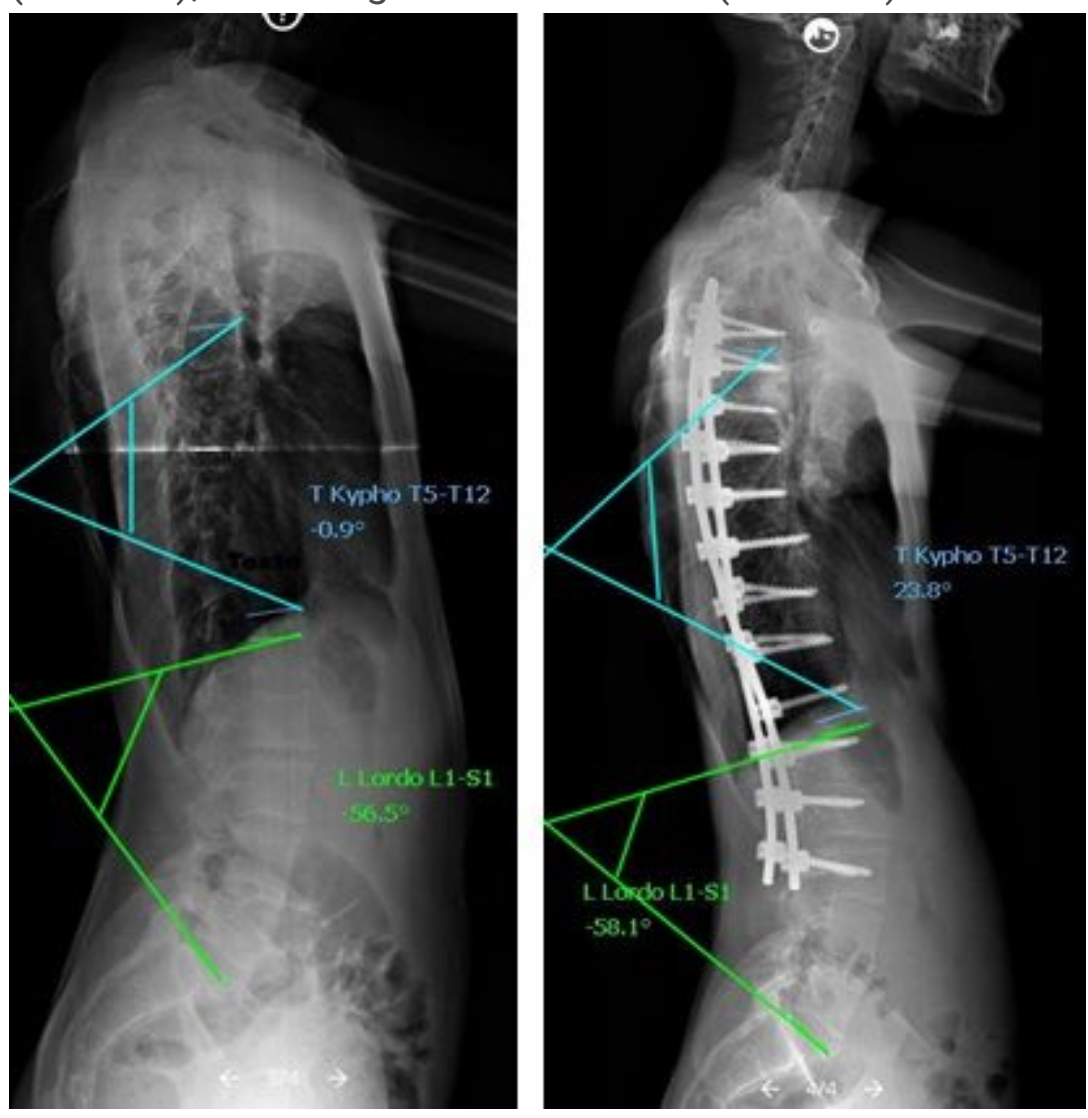
Figure 2

Demonstration of the increase in thoracic kyphosis (T Kypho T5-T12) and no improvement for the lumbar lordosis parameter (L Lordo L1-S1).

\section{Supplementary Files}

This is a list of supplementary files associated with this preprint. Click to download.

- Graphic1.jpg 